\title{
Modeling the Cost-effectiveness of Esophageal Cancer Screening in China
}

\author{
Yuanyuan $\mathrm{Li}^{1+}$, Lingbin $\mathrm{Du}^{2+}$, Youqing Wang ${ }^{2}$, Yuxuan $\mathrm{Gu}^{1}$, Xuemei Zhen ${ }^{1}$, Xiaoqian $\mathrm{Hu}^{1}$, Xueshan Sun \\ and Hengjin Dong ${ }^{*^{*}}$ (B)
}

\begin{abstract}
Background: This study aimed to examine the cost-effectiveness of one-time standard endoscopic screening with Lugol's iodine staining for esophageal cancer (EC) in China.

Methods: A Markov decision analysis model with eleven states was built. Individuals aged 40 to 69 years were classified into six age groups in five-year intervals. Three different strategies were adopted for each cohort: (1) no screening; (2) one-time endoscopic screening with Lugol's iodine staining with an annual follow-up for low-grade intraepithelial neoplasia (LGIN); and (3) one-time endoscopic screening with Lugol's iodine staining without follow-up. Qualityadjusted life-years (QALYS) indicated the effectiveness of the model. The incremental cost-effectiveness ratio (ICER) was used as the evaluation indicator. Sensitivity analysis was performed to assess the robustness of the model.

Results: One-time screening with follow-up was the undominated strategy for individuals aged 40-44 and 45-49 years, which saved USD 10,942.57 and USD 6611.73 per QALY gained compared to nonscreening strategy. For those aged 50-69 years, the nonscreening scenarios were undominated. One-time screening without follow-up was the extended dominated strategy. Compared to screening strategies without follow-up, all the screening strategies with follow-up were more cost-effective, with the ICER increasing from 299.57 USD/QALY for individuals aged 40-44 years to 1617.72 USD/QALY for individuals aged 65-69 years. Probabilistic sensitivity analysis (PSA) supported the results of the base case analysis.
\end{abstract}

Conclusions: One-time EC screening with follow-up targeting individuals aged 40-49 years was the most costeffective strategy.

Keywords: Esophageal cancer, Markov model, Screening, Cost-effectiveness analysis

\section{Background}

Esophageal cancer (EC) is a malignant tumor that still ranks as the ninth most common cancer and the sixth leading cause of cancer-related death worldwide $[1,2]$. There were 806,300 total cases, 472,500 new cases, and 436,000 deaths worldwide in 2017 [3]. EC incidence

\footnotetext{
*Correspondence: donghj@zju.edu.cn

${ }^{\dagger}$ Yuanyuan Li and Lingbin Du contributed equally to this article ${ }^{1}$ Center for Health Policy Studies, School of Public Health, Zhejiang University School of Medicine, 866 Yuhangtang Rd., 310058 Hangzhou, Zhejiang, China

Full list of author information is available at the end of the article
}

varies considerably according to geographical distribution, with over half of the worldwide incidence occurring in China [4]. The morbidity and mortality rates were 22.16 and 16.64 per 100,000 in 2013 in China [5]. Adenocarcinoma and esophageal squamous cell carcinoma (ESCC) are the major histological subtypes. Adenocarcinomas occur more frequently in developed countries, with an increasing trend in incidence, while ESCCs occur more frequently in less-developed regions [4]. ESCCs account for over $90 \%$ of all EC cases in China [6].

Several well-designed prospective preventive approaches have been developed to decrease the risk of EC; however, none have proven effective [7, 8]. Screening 
is a rapid, simple, and safe method that can be used to detect a disease in its early stage to reduce related risks and to treat the disease effectively. In the early 1980s, the WHO advocated secondary prevention strategies for early detection, diagnosis, and treatment. Cancer screening, as recommended by the $\mathrm{WHO}$, is the major strategy for primary and secondary disease prevention [9]. More than $90 \%$ of EC patients have progressed to an advanced stage at the time of diagnosis due to asymptomatic characteristics in the early stage, with a 5-year survival rate of $15-20 \%$ [10]. Screening can detect approximately $90 \%$ of early-stage EC cases and thus significantly improve the 5-year survival rate and also identify patients in the precancerous stage, an unstable state in which the patient could return to health through lifestyle interventions.

Currently, there are no global EC screening guidelines. However, national EC screening was first performed in 2005 in China [11]. In 2010, the EC screening program was conducted in Zhejiang Province and performed strictly following the national guidelines for the early detection of cancer [12]. Although it was used to screen an entire population, not all the individuals were included due to budget restrictions. A random cluster sampling method was used to identify the participants. A total of 29,762 residents aged 40-69 years from seven counties in Zhejiang Province were screened between 2010 and 2017. In brief, the screening procedures were performed as follows. Eligible residents aged 40 to 69 years were invited to receive health education specific to EC. Then, prospective participants voluntarily participated in the screening and provided their signed informed consent. Afterwards, they were recommended to undergo a physical examination before they completed a baseline structural questionnaire related to the risks of developing EC. Individuals who met the following criteria were excluded from the screening tests: severe respiratory disease, serious heart failure, abnormal coagulation function, aortic aneurysm, acute stage of corrosive inflammation of the upper digestive tract, severe abdominal distension, iodine allergy history, ascites, and severe spinal deformity. Participants meeting the clinical screening criteria were then examined by standard endoscopy accompanied by Lugol's iodine staining and indicative biopsy. All histological diagnoses were conducted by pathologists according to the AJCC cancer stage (seventh edition) [13]. Patients with clear histological diagnoses were recommended to undergo the following treatment. Patients with low-grade intraepithelial neoplasia (LGIN) were recommended to complete additional endoscopy screening in 1-3 years. Patients with intraductal carcinoma (IC) were recommended to undergo standard treatment (endoscopic submucosal dissection), while surgery was highly recommended for patients in the submucosal cancer (SM) stage. For patients with moderate-stage disease, surgery plus adjuvant chemoradiation was suggested, while patients with distant metastasis were treated with chemoradiation or treated for symptoms.

EC screening resulted in marked clinical benefits. First, the early diagnosis rates reached $70.59 \%$ to $98.1 \%$ [14]. In particular, a 10-year cohort study demonstrated decreased cumulative mortality $(3.35 \%$ vs $5.05 \%)$ and cumulative incidence $(4.17 \%$ vs $5.92 \%)$ in the screening population compared to the nonscreening population [15]. Moreover, the overall 5-year survival rate of patients with early EC reached $97.4-100 \%$, and the disease-specific 5-year survival rate reached 100\% [16-19]. However, the effectiveness of EC screening cannot be demonstrated without considering its economic value. An economic evaluation is useful, as it supports decision-making by providing an organized comparison of all available alternatives in terms of both their related costs and health outcomes [20]. Should the government launch a population-based EC screening program given the scarcity of medical resources and its relatively low incidence? Little is known about the cost and effectiveness of EC screening. This study aimed to compare the costs and effectiveness of one-time EC screening to identify the most cost-effective EC screening strategy and determine the age range for which screening should be performed in China.

\section{Methods \\ Model structure}

A decision analysis Markov model for EC with 11 health states was built with TreeAge Pro (2019). Only ESCC was modeled. The health states included normal, LGIN, IC, $\mathrm{SM}$, moderate cancer stage (Mod), advanced cancer stage (Adv), disease-free survival state of IC (DFS_IC), diseasefree survival state of SM (DFS_SM), disease-free survival state of moderate-stage cancer (DFS_Mod), progressionfree survival state of advanced-stage cancer (PFS_Adv), and death. Here, in the nonscreening cohorts, "normal health" was assumed to be the state of non-EC, while in the screening cohorts, it was assumed to be the state of health without LGIN and EC. IC included high-grade intraepithelial neoplasia, while moderate stage included stage IB, stage II, and stage III. Stage IV was classified as an advanced cancer stage. Overall, both IC and SM constituted the early EC stage, while moderate and advanced stages were identified as the invasive EC stage. Figure 1 summarizes the state transition processes, with the arrows presenting the transitions between states.

Individuals aged 40 to 69 years were assumed to be participants and classified into six age groups separated by 5 -year intervals (ages 40-44, 45-49, 50-54, $55-59,60-64$, and 65-69 years). Cohort simulation 


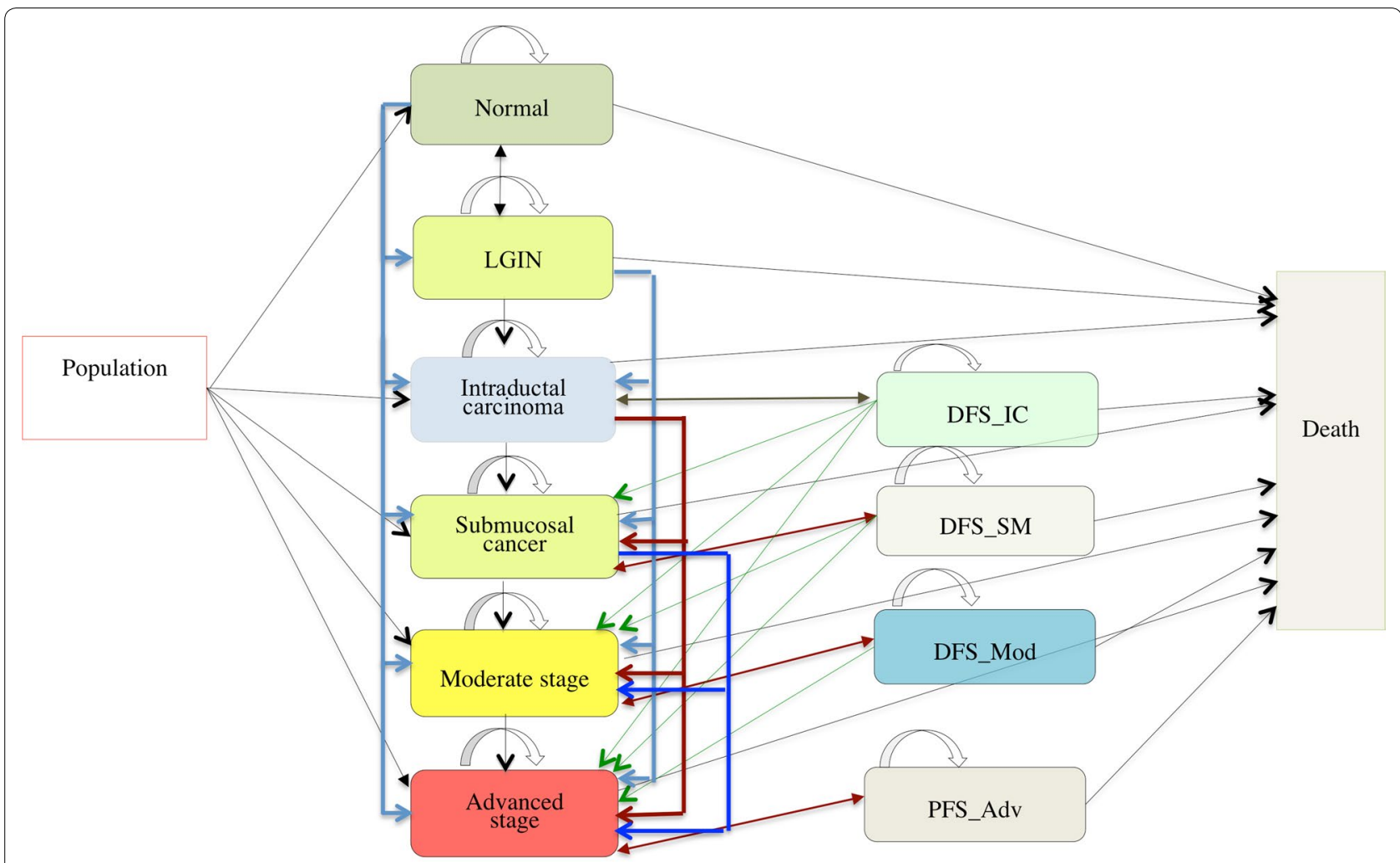

Fig. 1 Markov model of EC progression with 11 health states

was performed until the cohort age reached 79 years or until hypothetical death. The model assumed a 1-year cycle length. A hypothetical cohort was generated with 100,000 individuals assigned to each age group. Three different strategies were assessed for each cohort. (1) Nonscreening, a strategy that assumes that all the individuals were not screened, and no endoscopic follow-up for LGIN was conducted. Patients were not diagnosed until clinical symptoms appeared, which is referred to as passive treatment. Over $90 \%$ of the patients in these cohorts had progressed into moderate and advanced stages. (2) Screening with an annual follow-up for LGIN, a strategy that assumes all the individuals undergo one-time standard endoscopic screening, which could identify LGIN and EC. Approximately $90 \%$ of EC patients were diagnosed at an early stage. (3) Screening without follow-up for those with $L G I N$, a strategy that assumes all the individuals underwent one-time standard endoscopic screening, which could identify LGIN and EC. Most patients with EC were in the early stage. The study assumed that all the patients diagnosed by the three strategies had the correct diagnosis and that the treatment strategies adhered to standardized treatment scenarios, including the standardized clinical follow-up recommended by the
Guidelines for the Diagnosis and Treatment of EC 2018 in China.

\section{Effectiveness outcome}

Quality-adjusted life-years (QALYs) represented the effectiveness of the model, and the incremental costeffectiveness ratio (ICER) served as the economic evaluation indicator. The ICER indicates the additional cost per unit of additional effectiveness. The formula used to calculate the ICER was as follows: ICER $=\frac{\operatorname{Cost}_{A}-\operatorname{Cost}_{B}}{\mathrm{QALY}_{A}-\mathrm{QALY}_{B}}$. Cost-effectiveness analyses were used for comparisons between the competing strategies, including the "absolutely dominated strategy", an option that had both more costs and less effectiveness; the "extended dominated strategy", an option that was less costly and less effective than the alternative but had a higher ICER; and the "undominated best strategy", an option that was costeffective, with an ICER between 1 and 3 gross domestic products (GDPs) per capita based on the criterion recommended by the WHO [21]. Other outcomes assessed included costs, the cumulative incidence of $\mathrm{EC}$, and mortality. Moreover, the willingness-to-pay (WTP) was set as three times the GDP per capita (USD 51,000) in 2017 in China. 


\section{Cost and utility information}

Costs were estimated from a social perspective. The estimation consisted of screening costs, treatment costs, transportation, and wage loss of patients and relatives due to hospital visits. Screening costs were calculated using the data of the screening program in Zhejiang Province, while treatment costs were extracted from electronic medical records at Zhejiang Cancer Hospital. The average annual costs of the disease-free survival states were computed with the following formula: $\mathrm{C}=\mathrm{N} \times \sum_{1}^{\mathrm{i}} \mathrm{i} \times \mathrm{p}_{\mathrm{i}}$, where $\mathrm{C}$ is the annual costs of disease-free states, $\sum_{1}^{1} i \times p_{i}$ is the average cost per visit, $\mathrm{N}$ is the annual average number of hospital visits, $i$ is the number of physical examination items, and $\mathrm{p}$ is the price per item. $\mathrm{N}$ and $\mathrm{i}$ were suggested by the "Guidelines for the Diagnosis and Treatment of Esophageal Cancer 2018 in China”, and p was based on the price of medical services in provincial public hospitals in Zhejiang Province [22]. Transportation costs were computed using the second-class train or bus ticket price based on the distance between the visiting hospital and the patient's home. Wage losses were calculated by multiplying 1 day's income by the annual number of hospital visits (in days). We assumed that every patient had one accompanying relative. All costs were measured in the 2017 Chinese currency and were converted into US dollars using the purchasing power parity of 3.506 in 2017 [23]. All the items related to costs were assumed to inflate at the same inflation rate of $4.7 \%$ [24]. State-specific utilities were extracted from published papers [25-27]. A discount rate of $5 \%$ was used for both costs and effectiveness [28, 29]. Tables 1 and 2 display the state-specific costs and utilities, respectively.

Table 1 State-specific cost estimates for EC (USD)

\begin{tabular}{lcccc}
\hline State & Screening costs & $\begin{array}{l}\text { Treatment- } \\
\text { related costs }\end{array}$ & SA range & Distribution \\
\hline Normal & 60.3 & 0.0 & & \\
LGIN & 60.3 & 149.5 & & \\
IC & 60.3 & $17,561.9$ & $\pm 30 \%$ & Gamma \\
SM & 60.3 & $20,781.6$ & & \\
Mod & 60.3 & $25,217.4$ & & \\
Adv & 60.3 & $23,702.5$ & & \\
DFS_IC & 0.0 & 837.5 & & \\
DFS_SM & 0.0 & 1580.0 & & \\
DFS_Mod & 0.0 & 1580.0 & \\
PFS_Adv & 0.0 & 2873.0 & \\
Death & 0.0 & 0.0 & \\
\hline
\end{tabular}

SA one-way sensitivity analysis
Table 2 State-specific utilities for EC

\begin{tabular}{llllll}
\hline State & Mean & SD & SA range & Distribution & References \\
\hline Normal & 1.000 & 0.000 & $0.000-0.000$ & - & - \\
LGIN & 0.941 & 0.089 & $0.753-1.000$ & Beta & {$[26]$} \\
IC & 0.852 & 0.029 & $0.682-1.000$ & Beta & {$[25]$} \\
DFS_IC & 0.940 & 0.100 & $0.752-1.000$ & Beta & {$[27]$} \\
SM & 0.693 & 0.310 & $0.554-0.832$ & Beta & {$[25]$} \\
DFS_SM & 0.870 & 0.150 & $0.696-1.000$ & Beta & {$[27]$} \\
Mod & 0.780 & 0.140 & $0.624-0.936$ & Beta & {$[27]$} \\
DFS_Mod & 0.810 & 0.170 & $0.648-0.972$ & Beta & {$[27]$} \\
Adv & 0.720 & 0.180 & $0.576-0.864$ & Beta & {$[27]$} \\
PFS_Adv & 0.740 & 0.190 & $0.592-0.888$ & Beta & {$[27]$} \\
Death & 0.000 & 0.000 & $0.000-0.000$ & - & - \\
\hline SA one-way & & & &
\end{tabular}

SA one-way sensitivity analysis

\section{Data analysis}

\section{Probabilities in the initial cohort}

The probabilities for EC states in the initial nonscreening cohorts were calculated according to the 2012 agespecific incidence of EC in Zhejiang Province multiplied by the stage distribution at the time of diagnosis that was obtained from the hospital-based retrospective study [30]. The probabilities for EC states in the screening cohorts were computed by the age-specific EC detection rate multiplied by the stage distribution that was distinguished by screening. The initial probabilities of LGIN in the screening cohorts were the age-specific LGIN detection rate obtained from the screening program, while the initial probabilities in the nonscreening cohorts, were assumed to be zero since LGIN would not be diagnosed without screening. The probabilities for the normal state were one minus the sum of the probabilities for the other states for both scenarios. Table 3 displays the age-specific incidence and detection rate of EC and the age-specific detection rate of LGIN, while Table 4 displays the stage distributions for both scenarios.

\section{Transition probabilities between Markov states}

The annual incidence of EC was used to calculate the probabilities of transition from the normal state to the EC state for the nonscreening cohorts, while in the screening cohorts, the adjusted annual incidence of EC was used to compute the probabilities of transition. The formula used was $I_{a}=I_{p} \times R R$, where $I_{p}$ is the annual incidence of $E C$, $I_{a}$ is the adjusted annual incidence of EC, and RR is the annual incidence probability ratio that was computed with the formula used for the conversion between rate and probability using the cumulative incidence of ESCC in the screening group versus the nonscreening group $[15,31]$. Moreover, the stage distribution at the time of 
Table 3 Age-specific EC incidence, detection rate and mortality (per 100,000)

\begin{tabular}{lllllcc}
\hline Age & $\begin{array}{l}\text { EC annual } \\
\text { incidence }\end{array}$ & $\begin{array}{l}\text { EC annual } \\
\text { incidence for LGIN }\end{array}$ & EC detection rate & LGIN detection rate & $\begin{array}{l}\text { EC-related } \\
\text { mortality }\end{array}$ & All-cause mortality \\
\hline $40-44$ & 1.36 & 4.96 & 16.92 & 130.56 & 0.68 & 122.41 \\
$45-49$ & 4.90 & 17.68 & 70.65 & 545.00 & 2.19 & 184.60 \\
$50-54$ & 9.93 & 34.95 & 193.45 & 1492.33 & 7.57 & 343.55 \\
$55-59$ & 25.41 & 84.00 & 518.59 & 4000.54 & 14.87 & 475.18 \\
$60-64$ & 35.48 & 108.02 & 976.17 & 7530.48 & 22.03 & 738.42 \\
$65-69$ & 46.25 & 118.02 & 2072.48 & $-15,987.72$ & 33.08 & 1262.28 \\
$70-74$ & 63.97 & 163.22 & - & - & 53.42 & 2380.26 \\
$75-79$ & 64.45 & 164.36 & - & - & 65.73 & 4094.67 \\
\hline
\end{tabular}

One-way sensitivity analysis values $\pm 20 \%$; beta distribution was assumed for probability sensitivity analysis

Table 4 Stage distributions of EC under the screening and nonscreening scenarios

\begin{tabular}{llllllll}
\hline Stage & \multicolumn{2}{l}{ Nonscreening (\%) } & & \multicolumn{2}{l}{ Screening (\%) } & Distribution \\
\cline { 3 - 3 } & Proportion & $\begin{array}{c}\text { SA } \\
\text { range }\end{array}$ & & Proportion & $\begin{array}{l}\text { SA } \\
\text { range }\end{array}$ & \\
\hline IC & 3.65 & $2.92-$ & 88.24 & $70.59-$ & Dirichlet \\
& & 4.38 & & 100.00 & \\
SM & 4.93 & $3.94-$ & 2.52 & $2.02-$ & \\
& & 5.92 & & 3.02 & \\
Mod & 66.06 & $52.85-$ & 6.72 & $5.38-$ & \\
& & 79.27 & & 8.06 & \\
Adv & 25.36 & $20.29-$ & 2.52 & $2.02-$ & \\
& & 30.43 & & 3.02 & \\
\hline
\end{tabular}

SA one-way sensitivity analysis

diagnosis by passive treatment was used to identify EC states in both scenarios.

The probabilities of transferring from the LGIN state to the EC state were computed by the annual incidence of EC among the patients with LGIN (Table 3) multiplied by the EC stage distribution. The major difference was that the EC stage distribution was obtained from the results of screening programs for cohort with follow-up, while it was obtained from the results of passive treatment strategies without follow-up. In addition, the model assumed that a proportion of LGIN patients transferred to a normal state for strategies with follow-up [32-38], while no individuals transferred from an LGIN state to a normal state for strategies without follow-up. The incidence of EC among the LGIN patients was the adjusted incidence according to the risk ratio of the incidence of EC among LGIN patients compared to that among healthy subjects combined with the detected proportion of LGIN patients during screening. The risk ratio, which was summarized from published papers, was 3.66 [39-42]. No individuals were transferred from the LGIN state to the EC state under the nonscreening scenario since LGIN was not diagnosed due to asymptomatic characteristics without the implementation of screening. Other probabilities of transitioning between EC states were collected from various reports (Table 5 ).

The age-specific annual death probabilities for the normal state were defined as the difference between all-cause mortality and EC-related mortality. All-cause mortality was obtained from the sixth population service survey, while EC-related mortality was obtained from data on age-specific EC mortality in Zhejiang Province in 2012 $[30,43]$. LGIN was considered a precancerous lesion. The EC-specific 5-year survival rate was $100 \%$ for patients with IC and DFS_IC; therefore, people with LGIN or IC were not likely to die from EC. Consequently, the mortality rates for patients with LGIN, IC, and DFS_IC were assumed to be the same as those for patients in the normal state (Table 3). Death probabilities associated with SM and invasive cancer were identified from published papers, while the mortality risk was adjusted according to age (Table 3).

Moreover, a cycle length of 1 year was chosen; therefore, all the probabilities of transitions between states are presented as one-year probabilities. Given the different follow-up periods in the various data sources, we used two-step calculations. First, we converted the t-year follow-up probabilities into one-year rates. Then, we calculated one-year probabilities using one-year rates. The following formula was used to calculate the relationship between rate and probability. $\mathrm{r}=-\frac{\ln (1-\mathrm{p})}{\mathrm{t}} ; \mathrm{p}=1-\exp$ $(-r t)$, where $r$ indicates the rate, $p$ indicates the probability, and $t$ indicates the years of follow-up [31].

\section{Sensitivity analysis}

Sensitivity analyses for cost-effective screening strategies were performed. Probabilistic sensitivity analysis (PSA) permits the joint uncertainty across all the parameters in the model to be assessed at the same time, which involves sampling model parameter values from the distribution imposed on variables in the 
Table 5 Other parameters incorporated in the model

\begin{tabular}{|c|c|c|c|c|}
\hline Parameter & Input & SA range & Distribution & References \\
\hline \multicolumn{5}{|l|}{ Transition probability } \\
\hline Normal to LGIN & 0.0000 & - & - & - \\
\hline LGIN to normal & 0.1427 & $0.1142-0.1712$ & Beta & [32-38] \\
\hline IC to DFS_IC & 0.9363-d_nor & $0.7490-0.9363$ & Beta & {$[17]$} \\
\hline IC progress & 0.0534 & $0.0427-0.0641$ & Beta & {$[17,47-49]$} \\
\hline SM proportion & 0.2143 & $0.1714-0.2572$ & Dirichlet & Screening \\
\hline Mod proportion & 0.5714 & $0.4571-0.6857$ & Dirichlet & \\
\hline Adv proportion & 0.2143 & $0.1714-0.2572$ & Dirichlet & \\
\hline DFS_IC to IC & 0.0069 & $0.0055-0.0083$ & Beta & {$[50]$} \\
\hline DFS_IC progress & 0.0268 & $0.0214-0.0322$ & Beta & {$[51]$} \\
\hline SM proportion & 0.5556 & $0.4445-0.6667$ & Dirichlet & {$[51]$} \\
\hline Mod proportion & 0.4444 & $0.3555-0.5333$ & Dirichlet & {$[51]$} \\
\hline Adv proportion & 0.0000 & $0.0000-0.0000$ & Dirichlet & {$[51]$} \\
\hline SM to DFS_SM & 0.9051-d_nor & $0.7241-1.0000$ & Beta & {$[52]$} \\
\hline SM progress & 0.1386 & $0.1109-0.1663$ & Beta & {$[53,54]$} \\
\hline Mod proportion & 0.7562 & $0.6050-0.9074$ & Beta & {$[48,55-61]$} \\
\hline Adv proportion & 0.2438 & $0.1950-0.2926$ & Beta & {$[48,55-61]$} \\
\hline DFS_SM to SM & 0.0393 & $0.0314-0.0472$ & Beta & [62] \\
\hline DFS_SM progress & 0.0883 & $0.0706-0.1060$ & Beta & [53] \\
\hline Mod proportion & 0.7562 & $0.6050-0.9074$ & Beta & {$[48,55-61]$} \\
\hline Adv proportion & 0.2438 & $0.1950-0.2926$ & Beta & {$[48,55-61]$} \\
\hline Mod to DFS_Mod & 0.5930-d_nor & $0.4744-0.7116$ & Beta & {$[63]$} \\
\hline Mod to Adv & 0.0317 & $0.0254-0.0380$ & Beta & {$[64]$} \\
\hline DFS_Mod to Mod & 0.0425 & $0.0340-0.0510$ & Dirichlet & {$[64]$} \\
\hline DFS_Mod to Adv & 0.0097 & $0.0078-0.0116$ & Dirichlet & {$[64]$} \\
\hline Adv to PFS & 0.1967-d_nor & $0.1574-0.2360$ & Dirichlet & {$[65]$} \\
\hline PFS progress & 0.7002 & $0.5602-0.8402$ & Dirichlet & {$[66]$} \\
\hline RR & 0.7000 & $0.5600-0.8400$ & Lognormal & {$[15]$} \\
\hline \multicolumn{5}{|c|}{ EC state-specific death probability (age $\leq 65$ years) } \\
\hline SM & 0.0994 & $0.0795-0.1193$ & Beta & {$[67]$} \\
\hline DFS_SM & 0.0633 & $0.0506-0.0760$ & Beta & {$[67]$} \\
\hline Mod & 0.2988 & $0.2390-0.3586$ & Beta & {$[54]$} \\
\hline DFS_Mod & 0.1902 & $0.1522-0.2282$ & Beta & {$[54]$} \\
\hline Adv & 0.4613 & $0.3690-0.5536$ & Beta & {$[66,68]$} \\
\hline PFS & 0.4303 & $0.3442-0.5164$ & Beta & {$[66,68]$} \\
\hline \multicolumn{5}{|c|}{ Risk ratios of EC death probability among patients aged more than 65 years compared to patients aged less than 65 years } \\
\hline RR_SM/RR_DFS_SM & 1.30 & $1.20-1.50$ & Lognormal & {$[69]$} \\
\hline RR_Mod/RR_DFS_Mod & 1.20 & $1.10-1.30$ & Lognormal & [69] \\
\hline RR_Adv/RR_PFS_Adv & 1.16 & $1.10-1.20$ & Lognormal & [69] \\
\hline
\end{tabular}

SA one-way sensitivity analysis, $d \_$nor annual death probability for health individuals, which was defined as the difference between all-cause mortality and EC-related mortality

model. Initial cohort probabilities and death probabilities are assumed to be beta distributions, while the discount rate and inflation rate are considered triangular distributions. A gamma distribution was set for costs, and beta and Dirichlet distributions were set for transition probabilities. In addition, one-way sensitivity analyses were simulated because the impact of each parameter estimate varied independently and singly on the model results. The range of each parameter was assumed to be simply a "plausible" range. The following assumption was made. Initial probabilities, state transition probabilities, risk ratios, and health utility varied by $\pm 20 \%$ of the base case value, while costs varied by $\pm 30 \%$ of the base case value. Moreover, $0-8 \%$ was 
simulated for the discount rate, and 3.2-6.2\% was used for the inflation rate.

\section{Results}

\section{Simulated cumulative EC incidence and mortality}

Table 6 provides the details of the simulation. Compared to nonscreening, screening reduced the simulated cumulative EC incidence across all age groups. Strategies with follow-up reduced cumulative EC incidence more than strategies without follow-up. The reduction in cumulative EC incidence decreased with increasing screening age. Cumulative EC incidence was reduced by $29.9 \%$ to $18.9 \%$ and $29.6 \%$ to $2.0 \%$ among individuals aged 40 to 69 years in screening strategies with and without follow-up, respectively. Compared to screening strategies without follow-up, strategies with follow-up reduced cumulative EC incidence across all age groups. The reduction in cumulative EC incidence increased with increasing screening age. Cumulative EC incidence was reduced by $0.4 \%$ to $17.2 \%$ in individuals aged 40 to 69 years.

Compared to nonscreening, screening with follow-up for individuals aged 40-59 years resulted in a significant decrease in cumulative EC mortality but an obvious increase in cumulative EC mortality for individuals aged 60-69 years. The reduction in cumulative EC mortality varied from 28.8 to $4.9 \%$ among individuals between 40 and 59 years of age. Strategies without follow-up resulted in a considerable decrease in cumulative EC mortality for individuals 40-54 years of age but an obvious increase for individuals 55-69 years of age. The reduction decreased from 28.5 to $16.1 \%$ in individuals aged $40-44$ years to individuals aged 50-54 years. Compared to strategies without follow-up, all the strategies with follow-up resulted in a significant decrease in cumulative EC mortality. The reduction increased by $0.4 \%$ to $15.4 \%$ in individuals aged $40-44$ years to individuals aged $65-69$ years.

\section{ICERs}

The detailed results of the base case analyses are displayed in Table 7. Screening with follow-up was the undominated strategy, with USD 10,942.57 and USD 6611.73 saved per QALY gained for individuals aged 40-44 and 45-49 years, respectively, compared to nonscreening. For individuals aged 50-69 years, nonscreening was the undominated strategy. Screening without follow-up was the extended dominated strategy. Compared to screening strategies without follow-up, all the strategies using follow-up were cost-effective, with the ICER increasing from 299.57 USD/QALY for individuals aged 40-44 years to 1617.72 USD/QALY for individuals aged 65-69 years.

Table 6 Summary of simulated cumulative EC incidence and mortality among different screening strategies

\begin{tabular}{|c|c|c|c|c|c|c|c|}
\hline \multirow[t]{2}{*}{ Age } & \multirow[t]{2}{*}{ Strategy } & \multirow[t]{2}{*}{$\mathrm{Cl}($ per 100,000$)$} & \multirow[t]{2}{*}{ CM (per 100,000) } & \multicolumn{2}{|l|}{$\mathrm{RCl}(\%)$} & \multicolumn{2}{|l|}{ RCM (\%) } \\
\hline & & & & Scr* vs No_Scr & Scr_fol vs Scr_nfol & Scr* vs No_Scr & Scr_fol vs Scr_nfol \\
\hline \multirow[t]{3}{*}{$40-44$} & Non_scr & 1010.62 & 776.76 & - & - & - & - \\
\hline & Scr_nfol & 711.21 & 555.45 & -29.63 & - & -28.49 & - \\
\hline & Scr_fol & 708.61 & 553.32 & -29.88 & -0.37 & -28.77 & -0.38 \\
\hline \multirow[t]{3}{*}{$45-49$} & Non_scr & 1010.04 & 778.32 & - & - & - & - \\
\hline & Scr_nfol & 719.08 & 587.97 & -28.81 & - & -24.46 & - \\
\hline & Scr_fol & 708.81 & 579.22 & -29.82 & -1.43 & -25.58 & -1.49 \\
\hline \multirow[t]{3}{*}{$50-54$} & Non_scr & 994.97 & 766.49 & - & - & - & - \\
\hline & Scr_nfol & 726.70 & 642.84 & -26.96 & - & -16.13 & - \\
\hline & Scr_fol & 701.17 & 619.77 & -29.53 & -3.51 & -19.14 & -3.59 \\
\hline \multirow[t]{3}{*}{$55-59$} & Non_scr & 962.42 & 746.15 & - & - & - & - \\
\hline & Scr_nfol & 748.82 & 767.25 & -22.19 & - & 2.83 & - \\
\hline & Scr_fol & 691.03 & 709.45 & -28.20 & -7.72 & -4.92 & -7.53 \\
\hline \multirow[t]{3}{*}{$60-64$} & Non_scr & 857.84 & 649.89 & - & - & - & - \\
\hline & Scr_nfol & 720.35 & 829.78 & -16.03 & - & 27.68 & - \\
\hline & Scr_fol & 637.03 & 738.52 & -25.74 & -11.57 & 13.64 & -11.00 \\
\hline \multirow[t]{3}{*}{$65-69$} & Non_scr & 710.26 & 512.38 & - & - & - & - \\
\hline & Scr_nfol & 696.12 & 952.77 & -1.99 & - & 85.95 & - \\
\hline & Scr_fol & 576.12 & 806.34 & -18.89 & -17.24 & 57.37 & -15.37 \\
\hline
\end{tabular}

Scr_fol screening with follow-up, Scr_nfol screening without follow-up, Non_scr nonscreening, Scr* screening with or without follow-up, Cl cumulative EC incidence, $C M$ cumulative EC mortality, $R C /$ reduction in cumulative incidence compared to an alternative strategy, $R C M$ reduction in cumulative mortality compared to an alternative strategy 
Table 7 Summary of cost-effectiveness analyses among different EC screening strategies

\begin{tabular}{|c|c|c|c|c|c|}
\hline Age & Strategy & $\begin{array}{l}\text { Costs* (USD: } \\
\text { million) }\end{array}$ & QALYs (1000 years) & $\begin{array}{l}\text { ICER } \\
\text { Scr* vs No_Scr }\end{array}$ & $\begin{array}{l}\text { ICER } \\
\text { Scr_fol vs Scr_nfol }\end{array}$ \\
\hline \multirow[t]{3}{*}{$40-44$} & Non_scr & 40.02 & 1655.24 & - & - \\
\hline & Scr_nfol & 35.14 & 1655.59 & ED & - \\
\hline & Scr_fol & 35.17 & 1655.69 & $-10,942.57$ & 299.57 \\
\hline \multirow[t]{3}{*}{$45-49$} & Non_scr & 40.63 & 1557.40 & - & - \\
\hline & Scr_nfol & 38.65 & 1557.31 & ED & - \\
\hline & Scr_fol & 38.78 & 1557.68 & -6611.73 & 359.67 \\
\hline \multirow[t]{3}{*}{$50-54$} & Non_scr & 40.52 & 1437.30 & - & - \\
\hline & Scr_nfol & 44.85 & 1436.15 & $\mathrm{ED}$ & - \\
\hline & Scr_fol & 45.29 & 1437.07 & $A D$ & 471.63 \\
\hline \multirow[t]{3}{*}{$55-59$} & Non_scr & 39.83 & 1295.22 & - & - \\
\hline & Scr_nfol & 58.84 & 1291.50 & ED & - \\
\hline & Scr_fol & 60.30 & 1293.66 & $A D$ & 675.76 \\
\hline \multirow[t]{3}{*}{$60-64$} & Non_scr & 35.20 & 1120.73 & - & - \\
\hline & Scr_nfol & 70.82 & 1114.21 & $\mathrm{ED}$ & - \\
\hline & Scr_fol & 74.17 & 1117.47 & $A D$ & 1026.61 \\
\hline \multirow[t]{3}{*}{$65-69$} & Non_scr & 28.49 & 908.42 & - & - \\
\hline & Scr_nfol & 95.20 & 896.88 & $\mathrm{ED}$ & - \\
\hline & Scr_fol & 103.19 & 901.82 & $A D$ & 1617.72 \\
\hline
\end{tabular}

Scr_fol screening with follow-up, Scr_nfol screening without follow-up, Non_scr nonscreening, ICER incremental cost-effectiveness ratio, Scr* screening with or without follow-up, $A D$ absolutely dominated strategy, which was the option that had both more costs and less effectiveness, $E D$ extended dominated strategy, which was the option that was less costly and less effective than the alternative but had a higher ICER

Costs* stage and state-specific costs are displayed in eTable 1 and eTable 2

\section{Sensitivity analyses}

We ran 100,000 bootstrap interactions in the PSA. The primary results of the PSA are shown in Fig. 2. The findings revealed that considering a WTP of three times the GDP per QALY, screening with follow-up was the preferred strategy. The screening strategies without follow-up were dominated by the screening strategies with follow-up, with less costs and less QALYs than the nonscreening strategies. Figure 3 presents the differences in costs and QALYs per person between different screening strategies, along with a confidence ellipse that was computed using joint probability density. The confidence ellipse encompassed $95 \%$ of observations given the assumed WTP. More than $84 \%$ of the incremental costs

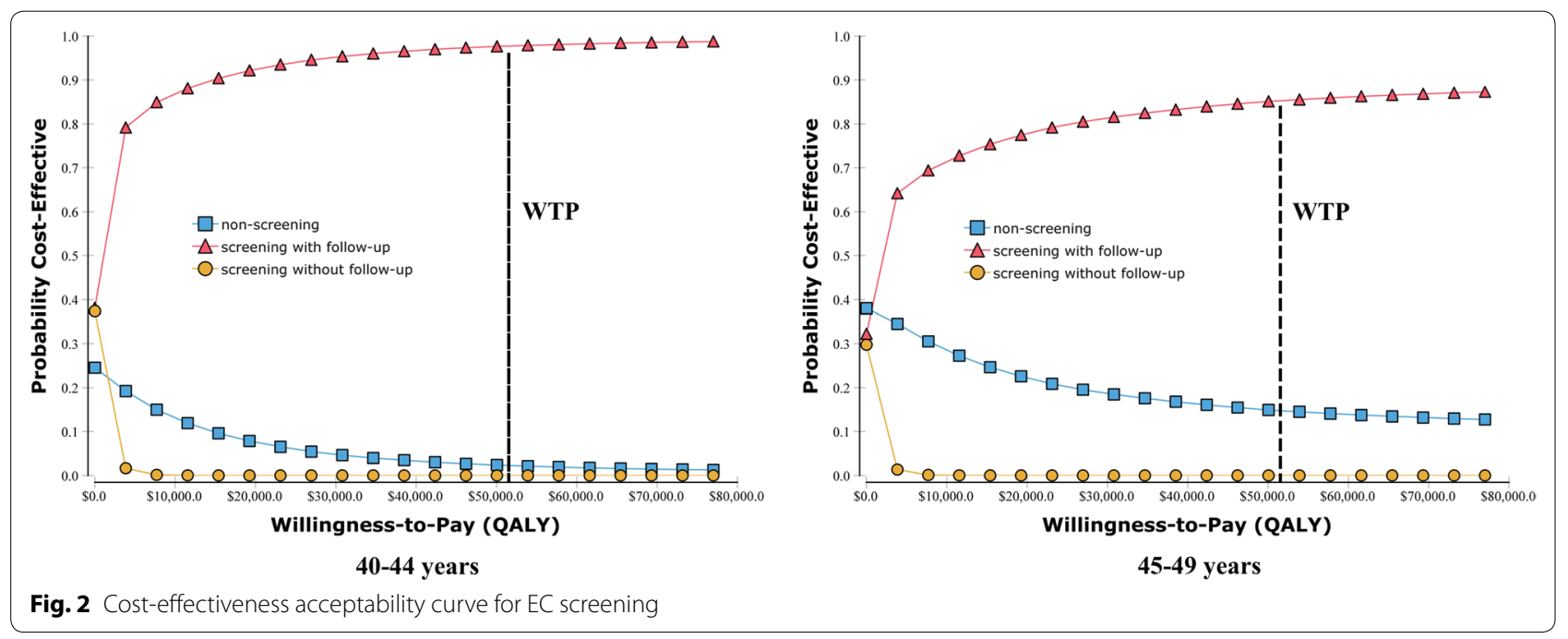




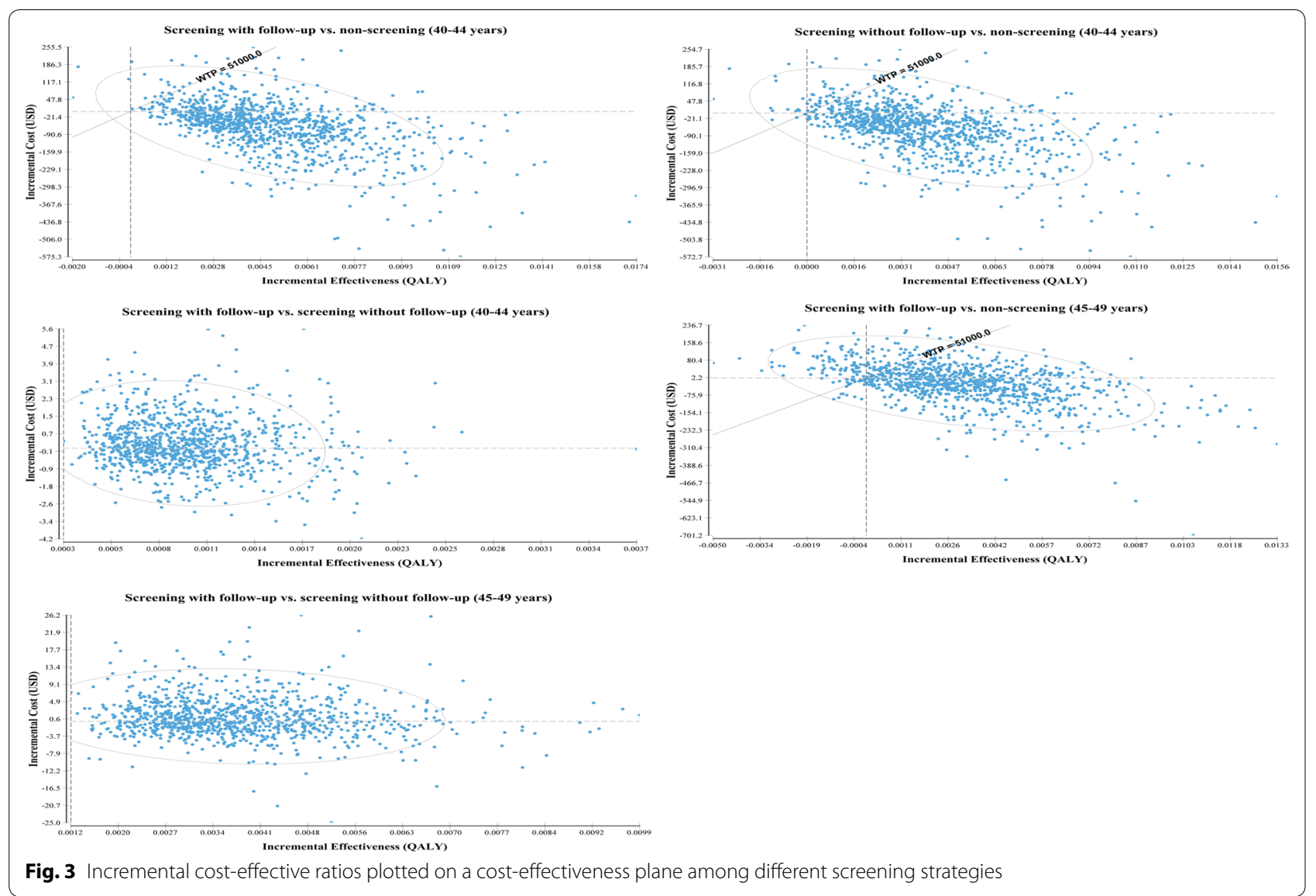

and effectiveness scatters of screening with follow-up over nonscreening are plotted in the south-east \& northwest quadrants, while all of the observations of screening with follow-up over screening without follow-up are plotted in the south-east and north-west quadrants. The outcome of the one-way sensitivity analysis is reported as a "tornado diagram" (Fig. 4), and only the parameters that accounted for $99 \%$ of the cumulative risk related to the ICER are displayed. The calculated ICER in the tornado diagram compared the screening with follow-up strategy to the nonscreening strategy. Figure 4 shows that the discount rate had a large impact on the ICER for individuals aged 40-44 years. An increase in the discount rate could reduce the cost-effectiveness of screening. However,

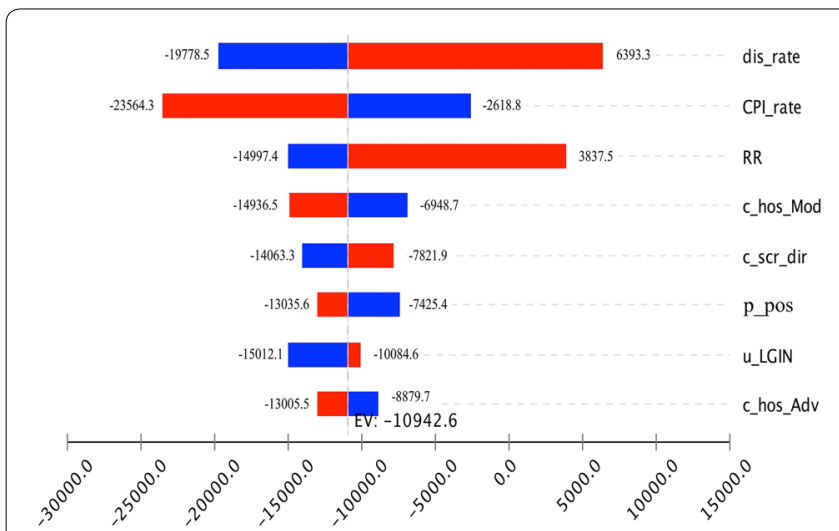

4-1 EC screening with follow-up compared to non-screening in age 40-44 years

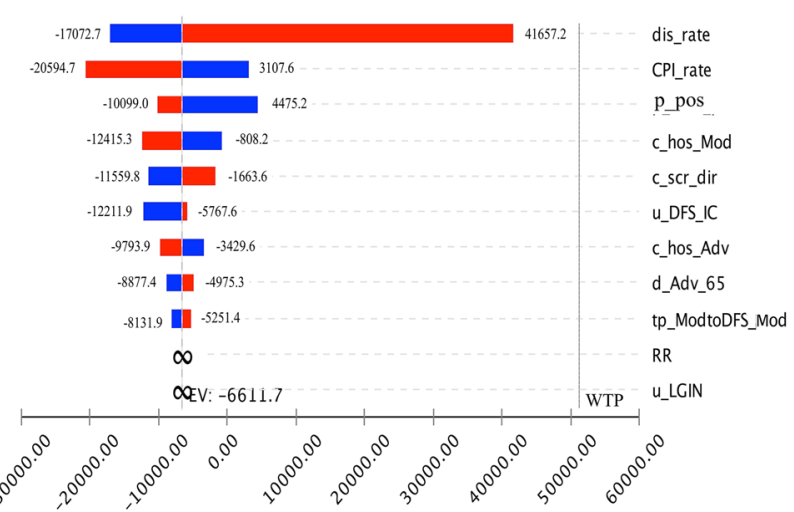

4-2 EC screening with follow-up compared to non-screening in age 45-49 years

Fig. 4 Tornado diagram assessing the effect of the uncertainty of a single parameter on the ICER (USD/QALY) 
even when the discount rate increased to $8 \%$, the ICER was less than the WTP. For individuals aged $45-49$ years, increasing the base case value of the RR to 0.84 and $u_{-}$ LGIN to 1 could make screening become the absolutely dominated strategy.

\section{Discussion}

This study was the first to examine the cost-effectiveness of population-based EC screening. A Markov decision analysis model was conducted based on the natural history of EC to compare the ICER of screening with or without follow-up to nonscreening. The model simulated the postoperative states, aligning well with the natural progression of EC, by assessing disease-free survival and progression-free survival for patients with early-, moderate-, and late-stage EC. Age was highly correlated with disease incidence and mortality. Hence, in this study, cohort simulation with six age groups separated by fiveyear intervals was performed until the cohort age reached 79 years or hypothetical death. Each age bracket was compared to its nonscreening group, which improved the comparability among strategies within age groups. In addition, the study assumed age-specific epidemiological assumptions, including age-specific initial probabilities, mortality, and transition probabilities. Furthermore, we calculated not only the direct medical costs but also the indirect costs using the human capital method.

Based on the criterion for cost-effectiveness recommended by the WHO [21], this study found that onetime EC screening targeting patients aged 40-49 years showed long-term cost-effectiveness. Though the data sources of EC incidence, detection, and cancer stage distributions derived from Zhejiang Province were used to calibrate the model parameters, the incidence and mortality in the screening areas in Zhejiang Province were quite comparable to those in China. Although Zhejiang-based costs were chosen, according to the one-way sensitivity analysis, a decrease or an increase by $30 \%$ of the costs obtained by screening with follow-up targeting patients with moderate- and advanced-stage EC aged 40-44 and 45-49 years still saved costs per QALY gained. Additionally, PSA confirmed that joint uncertainty across these parameters had no impact on the cost-effectiveness of screening targeting individuals in the same age group. Consequently, the results of this study suggest the cost-effectiveness of national one-time EC screening targeting individuals aged 40-49 years. Moreover, this screening should be performed with endoscopic followup, which enhances the benefits of screening for relatively small additive costs. Follow-up observation plays a key role in reducing EC mortality and morbidity. It not only promotes the transition of the LGIN state to a normal state through lifestyle interventions but also detects early-stage cancer and allows it to be treated early. Adenocarcinoma is the predominant histologic subtype in developed countries and shows an increasing trend in incidence. Our model simulated only the natural history of ESCC, and we cannot generalize the cost-effectiveness of ESCC screening to the whole world due to different natural histories and cost data. The cost-effectiveness of adenocarcinoma should be further studied.

In our model, cohort simulation was performed until the cohort age reached 79 years or hypothetical death, since life expectancy is 79 years in China. The results of the study showed that screening was more cost-effective in the younger population than in the older population. One-time EC screening targeting individuals aged 50-69 years was not cost-effective. The main reason for this finding was that screening targeting younger individuals resulted in a significant decrease in cumulative EC mortalities. Normal epithelial tissue takes decades to develop into cancer tissue based on its natural progression history. Early detection could result in early treatment, improved quality of life, and saved costs. Precancerous dysplasia is an unstable lesion, and early detection might be helpful for early intervention to reduce the risk of EC development. Screening in younger patients is more likely to detect $\mathrm{EC}$, especially precancerous lesions, in the early natural history progress. Moreover, older patients ae more likely to be diagnosed with other diseases or die of other diseases than younger patients. Treating older patients could increase costs and reduce quality of life due to increases in complications and infections. Moreover, treatment could result in an adverse psychological outcomes and mental stress. All of these findings suggest that it is not necessary to target individuals aged $50-69$ years for screening.

In 2016, the screening age was recommended to be extended to 74 years in the urban cancer screening program in China [44]. Although our study did not examine the economic value of EC screening in individuals aged more than 70 years, the results of the study imply that screening targeting elderly people is unlikely to be costeffective. However, EC screening targeting elderly individuals who are identified by the threshold of the risk function computed from the health risk appraisal questionnaire survey may have economic value. We strongly suggest that economically underdeveloped areas or areas at low risk of EC areas conduct a health risk appraisal survey for EC before clinical screening. Nevertheless, further studies should be performed to test these findings.

One-way sensitivity analyses revealed that the RR and u_LGIN had strong impacts on the cost-effectiveness of EC screening. Increasing the RR from 0.70 to 0.84 could make the screening strategy not cost-effective, indicating that screening is cost-effective only if it significantly 
reduces the incidence of EC. This result also confirmed the importance and necessity of endoscopic follow-up for LGIN, which could substantially reduce the risk of EC development. Furthermore, assuming that patients with LGIN had the same quality of life as healthy individuals, screening with follow-up targeting individuals aged 45-49 years was not cost-effective, with both higher costs and less QALYs. However, assuming that patients with LGIN had the same health status as healthy individuals was unlikely because patients with LGIN could have adverse psychological outcomes and mental stress when a true positive is detected, and these effects could have a negative impact on health.

Some limitations to this study should be considered when illustrating the outcomes. The main limitation was the data sources of EC incidence, detection, cancer stage distributions and costs derived from Zhejiang Province. However, the EC burden in Zhejiang Province could represent the national level very well. Although the current study analyzed Zhejiang-based costs, the sensitivity analyses confirmed the robustness of the model. In addition, our model simulation produced the long-term costs and effectiveness associated with the screening strategies. However, it did not provide accurate evaluations as do randomized clinical controlled trials. Furthermore, the current study may have underestimated the total expected costs because the hidden costs were not computed. However, this would not affect the identification of cost-effectiveness of EC screening, since the ICER was the indicator. Moreover, the sensitivity and specificity of the screening test were not studied since Lugol's iodine staining plus indicative biopsy is commonly regarded as the gold standard for EC diagnosis [45, 46].

\section{Conclusion}

One-time EC screening targeting individuals aged 40-49 years showed long-term cost-effectiveness in Zhejiang Province. The results of this study indicate the likely national cost-effectiveness of EC screening targeting the same age group. More importantly, endoscopic follow-up for LGIN should be performed to strengthen the screening benefit. For individuals over 50 years of age or from economically underdeveloped areas or areas at low risk of EC, the government and policymakers should take comprehensive measures to improve the opportunity for EC screening, including providing a health risk appraisal survey for EC. In addition, EC still poses a huge threat to the worldwide population, and building a solid global EC screening guideline and performing a cost-effectiveness analysis are currently crucial. Hopefully, much work on the topic of global EC screening will be conducted in the future.

\section{Supplementary information}

Supplementary information accompanies this paper at https://doi. org/10.1186/s12962-020-00230-y.

Additional file 1. Additional tables.

\section{Abbreviations}

EC: Esophageal cancer; ESCC: Esophageal squamous cell carcinoma; LGIN: Low-grade intraepithelial neoplasia; IC: Intraductal carcinoma; SM: Submucosal cancer; Mod: Moderate cancer stage; Adv: Advanced cancer stage; IC_DFS: Disease-free survival state of IC; SM_DFS: Disease-free survival state of SM; Mod_DFS: Disease-free survival state of moderate stage; Adv_PFS: Disease progress-free state of advanced stage; QALYs: Quality-adjusted life-years; ICER: Incremental cost-effectiveness ratio.

\section{Acknowledgements}

We would like to thank Alison Chang and Jasmine Lu from Princeton University, Jing Yu from Miami University, for their assistance in editing this paper. Conflicts of interest: The authors declare no conflicts of interest.

\section{Authors' contributions}

$Y L$ analyzed the data and wrote the manuscript. LD interpreted and analyzed the data. YW collected the data and acquired funding. YG drafted the work. XH performed the software analysis. XZ wrote the draft. XS revised the draft. HD designed the work and reviewed the draft. All authors read and approved the final manuscript.

\section{Funding}

This study was supported by the Medical Health Science and Technology Project of Zhejiang Provincial Health Commission (No. 2018251644).

\section{Availability of data and materials}

All data generated or analyzed during this study are included in this published article (Additional file 1).

\section{Ethics approval and consent to participate}

This article does not contain any studies with human participants or animals performed by any of the authors.

\section{Consent for publication}

Informed consent was not obtained because the study does not contain human participants.

\section{Competing interests}

The authors declare that they have no competing interests.

\section{Author details}

${ }^{1}$ Center for Health Policy Studies, School of Public Health, Zhejiang University School of Medicine, 866 Yuhangtang Rd., 310058 Hangzhou, Zhejiang, China. 2 Department of Cancer Prevention, Institute of Cancer Research and Basic Medical Science of Chinese Academy of Sciences, Cancer Hospital of University of Chinese Academy of Sciences, Zhejiang Cancer Hospital, 38 Banshan Guangqiao Rd., 310022 Hangzhou, Zhejiang, China.

Received: 7 February 2020 Accepted: 2 September 2020 Published online: 10 September 2020

\section{References}

1. Fitzmaurice $C$, Dicker D, Pain A, Hamavid H, Moradi-Lakeh M, et al. The global burden of cancer 2013. JAMA oncol. 2015;1(4):505-27.

2. Napier KJ, Scheerer M, Misra S. Esophageal cancer: a Review of epidemiology, pathogenesis, staging workup and treatment modalities. World J Gastrointest Oncol. 2014;6(5):112-20.

3. GBD 2017 Disease injury Incidence Prevalence Collaborators. Global, regional, and national incidence, prevalence, and years lived with disability for 354 diseases and injuries for 195 countries and territories, 
1990-2017: a systematic analysis for the Global Burden of Disease Study 2017. Lancet. 2018;392(10159):1789-858.

4. Arnold M, Soerjomataram I, Ferlay J, Forman D. Global incidence of oesophageal cancer by histological subtype in 2012. Gut. 2015;64(3):381-7.

5. Chen WQ, Zheng RS, Zhang SW, Zeng HM, Zuo TT, Xia CF, et al. Cancer incidence and mortality in China in 2013: an analysis based on urbanization level. Chin J Cancer Res. 2017;29(1):1-10.

6. Chinese Society of Gastroenterology gastrointestinal cancer group CSoDEedatgadpg. Expert consensus on china screening, diagnosis and treatment of early-stage and precancerous lesion of esophageal squamous cell carcinoma. Chin J Intern Med. 2016;55(1):73-85.

7. Reid BJ, Li X, Galipeau PC, Vaughan TL. Barrett's oesophagus and oesophageal adenocarcinoma: time for a new synthesis. Nat Rev Cancer. 2010;10(2):87-101.

8. Engel LS, Chow WH, Vaughan TL, Gammon MD, Risch HA, Stanford JL, et al. Population attributable risks of esophageal and gastric cancers. J Natl Cancer Inst. 2003;95(18):1404-13.

9. Hu GY, Dong P, Yan WQ, Xi Q. Discovery approach and economic burden of six kinds of common cancers patients in Beijing. Cancer Res Prev Treat. 2015;42(2):171-6.

10. Pennathur A, Gibson MK, Jobe BA, Luketich JD. Oesophageal carcinoma. Lancet (London, England). 2013;381(9864):400-12.

11. Dai M, Shi JF, Li N. Design and expected goal of early diagnosis and treatment of cancer project in Chinese cities. Chin J Prev Med. 2013;47(2):179-82.

12. Health disease Prevention and Control Bureau, Cancer Diagnosis and Treatment Project Expert Committee. Guideline for Early diagnosis and treatment of cancer. Beijing: Beijing People's Medical Publishing House; 2011

13. Stephen BE, David RB, Carolyn CC, April GF, Frederick LG, Yrotti A. AJCC cancer staging manual. Am Joint Committ Cancer. 2010;2010:103-15.

14. Li YY, Du LB, Hu XQ, Jaiswal S, Gu SY, Gu YX, et al. A suggested framework for conductiong esophageal cancer screening in China. J Dig Dis. 2019:19:722-9.

15. Wei WQ, Chen ZF, He YT, Feng H, Hou J, Lin DM, et al. Long-term followup of a community assignment, one-time endoscopic screening study of esophageal cancer in China. J Clin Oncol. 2015;33(17):1951-7.

16. Barret M, Prat F. Diagnosis and treatment of superficial esophageal cancer. Ann Gastroenterol. 2018;31(3):256-65.

17. Nagami Y, Ominami M, Shiba M, Minamino H, Fukunaga S, Kameda N, et al. The five-year survival rate after endoscopic submucosal dissection for superficial esophageal squamous cell neoplasia. Dig Liver Dis. 2017:49(4):427-33.

18. Pauthner M, Haist T, Mann M, Lorenz D. Surgical therapy of early carcinoma of the esophagus. Viszeralmedizin. 2015:31(5):326-30

19. Wang G, Hao C, Wei W. Longterm survival rate of precancerous lesions and early esophageal cancers after endoscopic mucosal resection. Chin J Digest Endosc. 2008:25(11):584-6.

20. Drummond MF, Sculpher MJ, Claxton K, Stoddart GL, Torrance GW. Methods for the economic evaluation of health care programmes. 4th ed. Oxford: Oxford University Press; 2015.

21. WHO-CHOICE. Cost-effectiveness threshold. http://www.who.int/choice/ costs/CER_thresholds/en/index.html. Accessed 16 March, 2012.

22. Health Commission of Zhejiang Province. Price of medical services in provincial public hospitals. http://www.zjwjw.gov.cn/art/2017/5/22/ art_1267707_452.html. Accepted 22 May 2017.

23. Organization for Economic Co-operation and Development(OECD) (2017) PPPs and exchange rates: purchasing power parities for GDP. https://statsoecdorg/Indexaspx?datasetcode=SNA_Table4. Accessed 16 Jul 2017.

24. National Bureau of Statistics of the People's Republic of China. Health service price index. http://www.stats.gov.cn/tjsj/ndsj/2018/indexch.htm.

25. Wang Y, Shi J, Du L, Huang H, Wang L, Zhu J, et al. Health-related quality of life in patients with esophageal cancer or precancerous lesions assessed by EQ-5D: a multicenter cross-sectional study. Thorac Cancer. 2020;11(4):1076-89.

26. Wang H, Pan Y, Guo C, Li F, Xu R, Liu M, et al. Health-related quality of life among rural residents aged 45-69 years in Hua County, Henan Province, China: results of ESECC Trial for esophageal cancer screening with endoscopy. Chin J Cancer Res. 2018;30(2):240-53.
27. Doherty MK, Leung Y, Su J, Naik H, Patel D, Eng L, et al. Health utility scores from EQ-5D and health-related quality of life in patients with esophageal cancer: a real-world cross-sectional study. Dis Esophagus. 2018:31:12.

28. Smith DH, Gravelle H. The practice of discounting in economic evaluations of healthcare interventions. Int J Technol Assess Health Care. 2001;17(2):236-43.

29. Committee of Chinese society of pharmaceutical economics. China Guidelines for Pharmacoecomonic Evaluations (2019 Edition).

30. Mao WM, Jin F, Yu M. Zhejiang cancer registry annual report. Hangzhou: Zhejiang Science and Technology Press; 2016.

31. Miller DK, Homan SM. Determining transition probabilities: confusion and suggestions. Med Decis Mak. 1994;14(1):52-8.

32. Whang SJ, Zhang LW, Wen DG, Li YS, Yu WF, Wang XL, et al. Analysis of endoscopic screening for the natural history of precancerous lesions in esophagus and cardia. Chian Cancer. 2007;34(7):370-3.

33. Wen D, Zhang L, Wang X, Li Y, Ma C, Liu X, et al. A 55-year surveillance of esophageal and gastric cardia precursors after a population-based screening in China. J Gastroenterol Hepatol. 2015;30(12):1720-5.

34. Wen DG, Zhang LW, Wang XL, Wen X, Yang Y, Chen YT, et al. Heterogeneity in esophageal and gastric cardia precursor progression during six-year endoscopic surveillance after population-based screening in a Chinese high-risk region. Thorac Cancer. 2017;8(4):328-36.

35. Chinese Academy of Medical Sciences, Esophageal Cancer Prevention and Treatment Research Team in Linxian. Outcome of esophageal epithelial hyperplasia: summary of follow-up by cytological examination. Cancer Res Prev Treat. 1974;1:42-6.

36. He YL, Zhang WJ, Liu YQ. Analysis of endoscopic screening for upper digestive tract cancer in Gaotai County, 2011-2015. China Cancer. 2017;26(6):447-51.

37. Lh L. Analysis of esophageal cancer/cardia cancer screening with iodine staining method throug endoscopy and esophageal atypical hyperplasia fellow-up in XianJu [Maser thesis]. Zhejiang: Zhejiang University; 2014.

38. Wang LD, Hq G. The development of esophageal epithelial hyperplasiasix years' follow-up of 66 subjects. J Henan Med Univ. 1991;26(4):328-30.

39. Dawsey SM, Lewin KJ, Wang GQ, Liu FS, Nieberg RK, Yu Y, et al. Squamous esophageal histology and subsequent risk of squamous cell carcinoma of the esophagus. A prospective follow-up study from Linxian, China. Cancer. 1994:74(6):1686-92.

40. Wang LD, Yang HH, Fan ZM, Lu XD, Wang JK, Liu XL, et al. Cytological screening and 15 years'follow-up (1986-2001) for early esophageal squamous cell carcinoma and precancerous lesions in a high-risk population in Anyang County, Henan Province, Northern China. Cancer Detect Prev. 2005;29(4):317-22.

41. Wang M. A prospective cohort study of the distribution and the progress rate of precancerous lesions of Eesophageal squamous cell carcinoma in high-risk areas of china. Chinese academy of medical sciences, Peking union medical college Master's thesis. 2016.

42. Tao DM, Xu YZ, Gu YK, Wang DY, Wang XH, Gh Y. A study of the carcinogenesis time and incidence of carcinoma in 46161 cases with normal and Hyperplastic esophageal epithelia. Cancer Prev Res. 1997;1997:155-6.

43. Population Census Office of the State Council, Population Statistics Department of National Bureau of Statistics. Tabulation on the 2010 population census of the people's republic of China. 2012. http://www. stats.gov.cn/tjsj/pcsj/rkpc/6rp/indexch.htm. Accessed 1 Dec 2012.

44. Chen WQ, Li N, Shi JF, Chen HD, Li J, et al. Progress of cancer screening program in urban China. China Cancer. 2019;28(1):23-5.

45. Dawsey SM, Fleischer DE, Wang GQ, Zhou B, Kidwell JA, Lu N, et al. Mucosal iodine staining improves endoscopic visualization of squamous dysplasia and squamous cell carcinoma of the esophagus in Linxian, China. Cancer. 1998;83(2):220-31.

46. Hashimoto CL, Iriya K, Baba ER, Navarro-Rodriguez T, Zerbini MC, Eisig JN, et al. Lugol's dye spray chromoendoscopy establishes early diagnosis of esophageal cancer in patients with primary head and neck cancer. Am J Gastroenterol. 2005;100(2):275-82.

47. Nakagawa K, Koike T, lijima K, Shinkai H, Hatta W, Endo H, et al. Comparison of the long-term outcomes of endoscopic resection for superficial squamous cell carcinoma and adenocarcinoma of the esophagus in Japan. Am J Gastroenterol. 2014;109(3):348-56.

48. Yamashina T, Ishihara R, Nagai K, Matsuura N, Matsui F, Ito T, et al. Long-term outcome and metastatic risk after endoscopic resection of 
superficial esophageal squamous cell carcinoma. Am J Gastroenterol. 2013;108(4):544-51.

49. Tsuji Y, Nishida T, Nishiyama O, Yamamoto K, Kawai N, Yamaguchi S, et al. Clinical outcomes of endoscopic submucosal dissection for superficial esophageal neoplasms: a multicenter retrospective cohort study. Endoscopy. 2015;47(9):775-83.

50. Daoud DC, Suter N, Durand M, Bouin M, Faulques B, von Renteln D. Comparing outcomes for endoscopic submucosal dissection between Eastern and Western countries: a systematic review and meta-analysis. World J Gastroenterol. 2018;24(23):2518-36.

51. Konda VJ, Ross AS, Ferguson MK, Hart JA, Lin S, Naylor K, et al. Is the risk of concomitant invasive esophageal cancer in high-grade dysplasia in Barrett's esophagus overestimated? Clin Gastroenterol Hepatol. 2008;6(2):159-64.

52. Rice TW, Lerut TE, Orringer MB, Chen LQ, Hofstetter WL, Smithers BM, et al. Worldwide Esophageal Cancer Collaboration: neoadjuvant pathologic staging data. Dis Esoph. 2016;29(7):715-23.

53. Gockel I, Sgourakis G, Lyros O, Polotzek U, Schimanski CC, Lang H, et al. Risk of lymph node metastasis in submucosal esophageal cancer: a review of surgically resected patients. Expert Rev Gastroenterol Hepatol. 2011;5(3):371-84.

54. Chan DS, Reid TD, Howell I, Lewis WG. Systematic review and metaanalysis of the influence of circumferential resection margin involvement on survival in patients with operable oesophageal cancer. Br J Surg. 2013;100(4):456-64.

55. Chen SB, Weng HR, Wang G, Yang JS, Yang WP, Liu DT, et al. Surgical treatment for early esophageal squamous cell carcinoma. Asian Pac J Cancer Prev. 2013;14(6):3825-30.

56. Fujita H, Sueyoshi S, Yamana H, Shinozaki K, Toh U, Tanaka Y, et al. Optimum treatment strategy for superficial esophageal cancer: endoscopic mucosal resection versus radical esophagectomy. World J Surg. 2001;25(4):424-31.

57. Stein HJ, Feith M, Mueller J, Werner M, Siewert JR. Limited resection for early adenocarcinoma in Barrett's esophagus. Ann Surg. 2000;232(6):733-42.

58. Mitsuo T, Hiroshi Y, Shoichi K, Naoki H, Dipok KD, Shun'ichi A, et al. Clinicopathological features of superficial squamous cell carcinoma of the esophagus. Am J Surg. 1997;174:49-53.

59. van Sandick JW, van Lanschot JJ, ten Kate FJ, Offerhaus GJ, Fockens P, Tytgat GN, et al. Pathology of early invasive adenocarcinoma of the esophagus or esophagogastric junction: implications for therapeutic decision making. Cancer. 2000;88(11):2429-37.

60. Yoshinaka H, Shimazu H, Fukumoto T, Baba M. Superficial esophageal carcinoma: a clinicopathological review of 59 cases. Am J Gastroenterol. 1991;86(10):1413-8.

61. Natsugoe S, Baba M, Yoshinaka H, Kijima F, Shimada M, Shirao K, et al. Mucosal squamous cell carcinoma of the esophagus: a clinicopathologic study of 30 cases. Oncology. 1998;55(3):235-41.

62. Sgourakis G, Gockel I, Lang H. Endoscopic and surgical resection of T1a/ T1 b esophageal neoplasms: a systematic review. World J Gastroenterol. 2013;19(9):1424-37.

63. Karstens KF, Izbicki JR, Reeh M. Does the margin matter in esophageal cancer. Dig Surg. 2018;35(3):196-203.

64. Knight WRC, Zylstra J, Wulaningsih W, Van Hemelrijck M, Landau D, Maisey $\mathrm{N}$, et al. Impact of incremental circumferential resection margin distance on overall survival and recurrence in oesophageal adenocarcinoma. BJS Open. 2018;2(4):229-37.

65. Zou ZH, Liao XZ, Xu KK, Shi ZH, Zhu SL, Hf X. Analysis of screening results of 6400 urban lung cancer in high-risk groups. Pract Prev Med. 2016;23(5):593-4

66. Saddoughi SA, Reinersman JM, Zhukov YO, Taswell J, Mara K, Harmsen SW, et al. Survival after surgical resection of stage IV esophageal cancer. Ann Thorac Surg. 2017;103(1):261-6.

67. Rice TW, Ishwaran H, Hofstetter WL, Kelsen DP, Apperson-Hansen C, Blackstone $\mathrm{EH}$, et al. Recommendations for pathologic staging (pTNM) of cancer of the esophagus and esophagogastric junction for the 8th edition AJCC/UICC staging manuals. Dis Esoph. 2016;29(8):897-905.

68. U.S. National cancer Institution(2019). Cancer Stat Fact Sheets: Esophageal Cancer. http://seer.cancer.gov/statfacts/html/esoph.html.

69. Chen ZL, Ren YH, Du XLL, Yang J, Shen YW, Li ST, et al. Incidence and survival differences in esophageal cancer among ethnic groups in the United States. Oncotarget. 2017;8(29):47037-51.

\section{Publisher's Note}

Springer Nature remains neutral with regard to jurisdictional claims in published maps and institutional affiliations.
Ready to submit your research? Choose BMC and benefit from:

- fast, convenient online submission

- thorough peer review by experienced researchers in your field

- rapid publication on acceptance

- support for research data, including large and complex data types

- gold Open Access which fosters wider collaboration and increased citations

- maximum visibility for your research: over 100M website views per year

At BMC, research is always in progress.

Learn more biomedcentral.com/submissions 